\title{
Study on Adsorption and Separation of Gas in Metal-organic Framework Materials Based on Density Functional Theory
}

\author{
Du Zhehua ${ }^{1, *}$, Lin $\mathrm{Xin}^{2}$ \\ ${ }^{1}$ Wuhan Second Ship Design and Research Institute, 430205 Wuhan, China \\ ${ }^{2}$ Hubei Province Engineering Consulting Co., LTD., 430071 Wuhan, China
}

\begin{abstract}
This article reviews the recent progress on predicting the adsorption properties of metal-organic framework by using classical density functional theory and focused on the application of the classical density functional theory to the high-throughput screening, which is accelerated by fast Fourier Transform. Comparing to the conventional molecular simulations, the advantage of the accelerated classical density functional theory is the calculation speed, especially for simple small molecule systems, which makes the high-throughput screening on MOF materials feasible. However, it appears that there is a lack of efficient method to deal with the complicated molecules. How to construct a reasonable free energy functional of complicated fluid is the main challenge to state of art classical density functional theory. In a word, the improvement of CDFT theory and the combination of CDFT and molecular simulation are the two main ways for CDFT to predict gas adsorption in MOF.
\end{abstract}

\section{Introduction}

Metal organic framework (MOF for short) is a coordination compound with three-dimensional porous structure based on metal ions or metal oxides as the nodes. Since it was synthesized in the 1990s, it has been considered to have a good potential application prospect in the fields of adsorption separation, catalysis and sensing detection due to its excellent properties. To date, thousands of metal-organic skeleton materials have been synthesized and billions of hypothetical MOF material structures have been proposed [1]. The reason why MOF materials are considered to have a wide range of application prospects lies in their excellent adsorption properties. Therefore, the prediction of the adsorption characteristics of MOF materials is of vital significance in the synthesis and application.

The traditional method to predict the adsorption characteristics of MOF materials is through computer simulation. Computer simulation is currently the most accurate method for predicting MOF adsorption. However, due to its huge computational workload and low efficiency, it is limited in practical applications. It is imperative to develop more efficient calculation methods to predict the adsorption properties of MOF materials. Classical density functional theory (CDFT for short) seems to be a better choice. Under the premise of ensuring the same calculation accuracy, CDFT can often show higher calculation efficiency than computer simulation. It is expected to be a theoretical method to replace computer simulation in some special occasions.

\section{Classical density functional theory of gas adsorption in MOF materials}

The basic idea of classical density functional theory is to solve fluid density distribution through the variational method based on thermodynamic equilibrium condition - free energy (closed system) or giant potential (open system) minimum principle, and then obtain other physical quantities of system. In this process, how to construct the functional form of free energy $F$ or giant potential $\omega$ on fluid density distribution is the core issue. Generally speaking, free energy is divided into the sum of ideal gas, external field action and the residual contribution of fluid molecular action.

$$
F=F^{i d}+F^{e x t}+F^{e x}
$$

In the formula, $F_{i d}$ is the ideal free energy of adsorbed gas, that is, the fluid contribution of kinetic energy term. $F_{\text {ext }}$ is the contribution of external field to free energy, that is, the contribution of the interaction between fluid and external field. $F_{e x}$ is the residual free energy, which is the contribution of the interaction between fluid molecules.

Based on the related theory of statistical mechanics, ideal free energy can be accurately expressed by fluid density distribution functional form of $\rho(r)$.

$$
F^{i d}[\rho(r)]=k_{B} t \int \rho(r)\left[\ln \rho(r) \Lambda^{3}-1\right] d r
$$

In the formula, $\Lambda$ is de Broglie wavelength. $k_{B}$ is Boltzmann constant. $T$ is the temperature.

The contribution of external field action to free energy is considered by external conditions, such as

\footnotetext{
* Corresponding author: shunli878@163.com
} 
external electric field, confined space and so on. If external conditions are not affected by fluid feedback, this contribution can be simply expressed as the following equation. In the equation, $V^{\text {ext }}(r)$ is external potential field.

$$
F^{e x t}[\rho(r)]=\int \rho(r) V^{e x t}(r) d r
$$

Residual free energy $F^{e x}$ reflects the complex interactions between molecules. Generally, there is no precise expression and only approximate methods can be used. Generally, residual free energy is divided into the sum of the contributions of hard ball repulsion and attraction. Different approximations are used for these two parts.

$$
F^{e x}=F_{h s}^{e x}+F_{\text {attr }}^{e x}
$$

Since classical density functional theory was proposed in the $1970 \mathrm{~s}$, it has long been applied to systems with highly symmetrical geometric structures, such as slit-shaped channels and cylindrical channels. However, the systems that have been applied to a threedimensional complex spatial structure, such as MOF discussed in this article, have only recently been developed.

In 2009, Siderius et al. [2] applied CDFT to adsorption study of MOF materials. They studied the adsorption phenomenon of $\mathrm{H}_{2}$ in MOF-5 at high temperature, and got the results in agreement with experiments and simulations. Liu et al. [3] improved the method so that CDFT can accurately predict the adsorption characteristics of MOF materials in the full temperature range. Both Siderius and Liu predicted that the best adsorption site for $\mathrm{H}_{2}$ in MOF-5 is near metal ions, as shown in Figure 1.

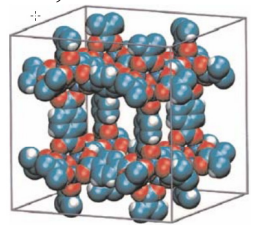

(a)IRMOF-1

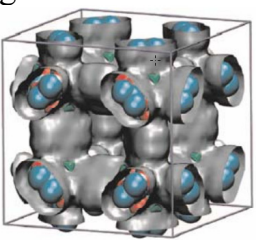

(b) $p=7.95 \mathrm{MPa}$

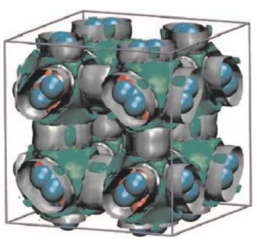

(c) $p=17.9 \mathrm{MPa}$
Fig. 1. Density profiles of $\mathrm{H}_{2}$ in MOF-5 (isosurface)

\section{Large-scale screening of adsorbent materials}

\subsection{Applications of fast Fourier transform}

When classical density functional theory is used to predict the adsorption of gas and its mixture by MOF material, density distribution equation needs to be solved numerically in three dimensions. Due to the complex three-dimensional pore structure of MOF materials, the pore space needs to be divided into different calculation grid points according to the specific structure of different MOF materials, which requires a large amount of calculation. To solve this problem, Wu et al. [4] introduced fast Fourier transform method. The calculation of residual free energy $F_{e x}$ is the most timeconsuming part when CDFT is used to deal with the density distribution with complex three-dimensional structure. The residual free energy can be expressed as follows.

$$
\begin{aligned}
& F^{e x}=\int \rho(r) f[\bar{\rho}(r)] d r \\
& \bar{\rho}(r)=\int \rho(r) w\left(r-r^{\prime}\right) d r
\end{aligned}
$$

By Fourier transform, equation (6) can be reduced to the following equation in Fourier space. By Fourier transform, equation (6) can be reduced to the following equation in Fourier space. Where $\rho(k)$ is the Fourier transform of the function $\rho(r)$.

$$
\bar{\rho}(k)=\rho(k) \rightsquigarrow(k)
$$

Since the computational complexity of fast Fourier transform is only $N^{D} \log _{2} N^{D}$, the use of equation (7) instead of equation (6) can greatly improve the computational efficiency.

The introduction of fast Fourier transform makes CDFT a more efficient tool for predicting the adsorption performance of MOF materials, and greatly expands the application range of CDFT. One of the most important applications is the large-scale screening of MOF adsorption materials.

\subsection{Large-scale screening}

There are many different types of MOF materials, and hundreds of millions of structures can be generated through the permutation and combination of different point groups, different metal ion nodes, and organic linkers. Nowadays, billions of hypothetical MOFs have been proposed. It is obviously unrealistic to adopt experimental methods, and the concept of "select first, then synthesize" must be adopted. First, a round of theoretical screening of these optional MOF materials is carried out to find a few of most potential structures, and then only these few structures are synthesized and tested. In this process, the screening method is crucial, which must be both accurate and efficient.

The application of CDFT in the large-scale screening of MOF adsorption materials was originally completed by $\mathrm{Wu}$ et al. [4]. They examined adsorption properties of hydrogen and methane in 1,200 MOF materials, respectively, and obtained highly consistent results with computer simulations, as shown in Figure 2.

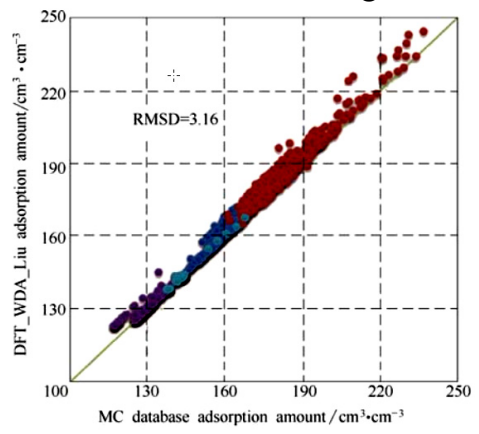

(a) $243 \mathrm{~K}, 10 \mathrm{MPa}$ 


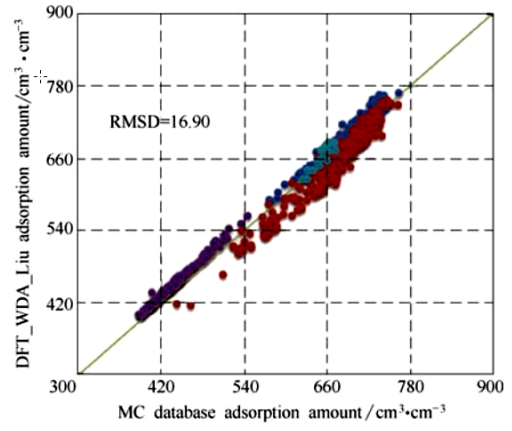

(b) $77 \mathrm{~K}, 5 \mathrm{MPa}$

Fig. 2. Comparison between CDFT and MC prediction of $\mathrm{H}_{2}$ adsorption in 1200 types MOF materials

$\mathrm{Wu}$ et al. approximated hard ball term, and respectively used 4 different approximation methods to calculate the contribution of attraction term. The results show that the results of four methods are indistinguishable at high temperature, and Liu's WDA is the most accurate at low temperature. The results also show that the equation state quality has a vital influence on the results of calculation. As shown in Fig. 3, the prediction result of the more accurate MBWR (Modified Benedict-Webb-Rubin) state equation is significantly better than FMSA(First-Order Mean Spherical Approximation).

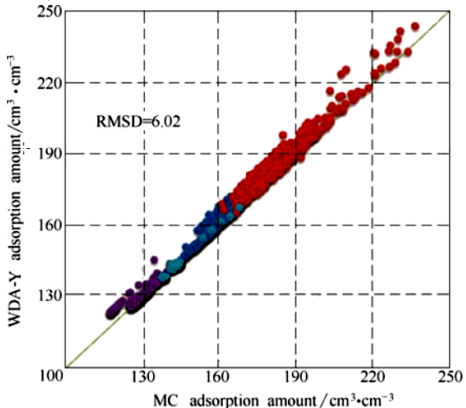

(a) MBWR

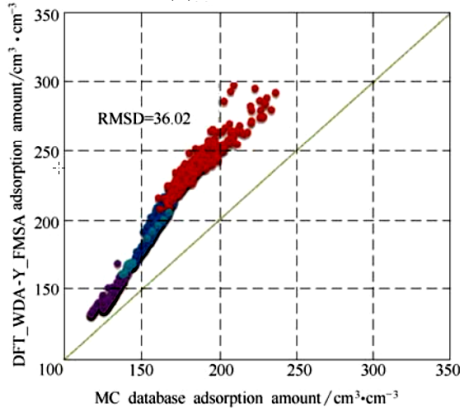

(b) FMSA

Fig. 3. Effect of different equation of state on predicting Results

Liu et al. [5] further expanded the scope of prediction and predicted the adsorption characteristics of $712 \mathrm{MOF}$ materials under 441 different temperature and pressure conditions, and screened the best hydrogen storage MOF materials under different external conditions. According to the calculation results of Liu et al. (see Figure 4), under the conditions of $77 \mathrm{~K}$ and $10 \mathrm{MPa}$, the amount of $\mathrm{H}_{2}$ adsorbed by MOF can reach $37 \mathrm{~mol} \cdot \mathrm{L}^{-1}$, even exceeding the density of liquid hydrogen $\left(35 \mathrm{~mol} \cdot \mathrm{L}^{-1}\right)$. However, at high temperature, the hydrogen storage performance of MOF material did not improve substantially, which was still below $5 \mathrm{~mol} \cdot \mathrm{L}^{-1}$.

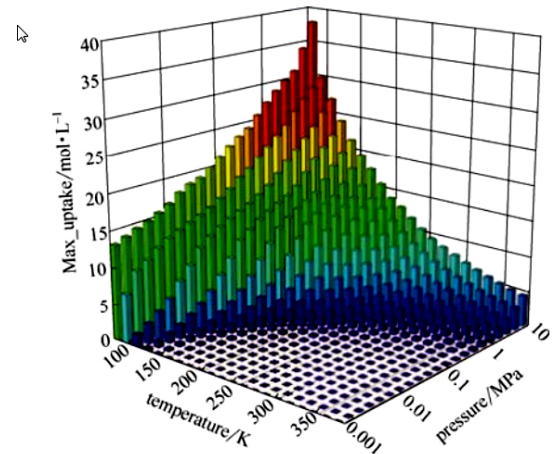

Fig. 4 Highest adsorption amount for 712 types of MOF material on different temperatures and pressures

Recently, Liu et al. [8] further applied the CDFT method of predicting MOF adsorption to the field of adsorption desulfurization. Although thiophene sulfides involved in this work exist in liquid phase, their behavior is basically consistent with gas molecules at low densities. This work shows that CDFT can not only predict adsorption characteristics of small molecule gas in MOF, but also predict adsorption characteristics of more complex molecules such as thiophene sulfide. Similar to the prediction of small molecule gas, the best desulfurization adsorbents for different sulfur concentrations were selected through large-scale CDFT calculation, which can provide guidance for the experimental synthesis of new adsorption desulfurization materials.

\section{Prediction of diffusion coefficient}

In addition to the adsorption capacity and selectivity, the adsorption and desorption rate is also an important index to evaluate the properties of MOF materials when they are used for gas adsorption separation. Diffusion coefficient is an extremely critical index for investigating the adsorption kinetic properties of MOF materials. Generally speaking, the larger the diffusion coefficient, the higher the absorption and desorption rate. The prediction of gas diffusion coefficient in porous materials is currently mainly completed by molecular dynamics simulation [6]. However, similar to the predicted adsorption capacity, speed is still a shortcoming of computer simulation. Especially for the prediction of diffusion coefficient, the computational resources consumed by simulation will be much greater than the predicted adsorption capacity.

Although CDFT cannot directly predict the diffusion coefficient as a kinetic physical quantity, it can be linked with the diffusion coefficient by the residual entropy scale theory [7]. Residual entropy scaling theory gives the universal relation between diffusion coefficient and residual entropy.

$$
D^{*}=A \exp \left(B s^{e x^{*}}\right)
$$

In the formula, $D^{*}$ is diffusion coefficient after dimension $1 ; s^{e x *}$ is residual entropy after dimension $1 ; A$ and $B$ are universal constants.

The theory of residual entropy scaling establishes a bridge between thermodynamic properties and kinetic parameters, so that people can predict dynamic diffusion 
coefficient by calculating residual entropy of thermodynamics. Recently, Liu et al. [8] used CDFT to calculate residual entropy and successfully predicted diffusion coefficient in MOF materials by combining Knudsen's theory and mean free volume theory (see Fig. 5).

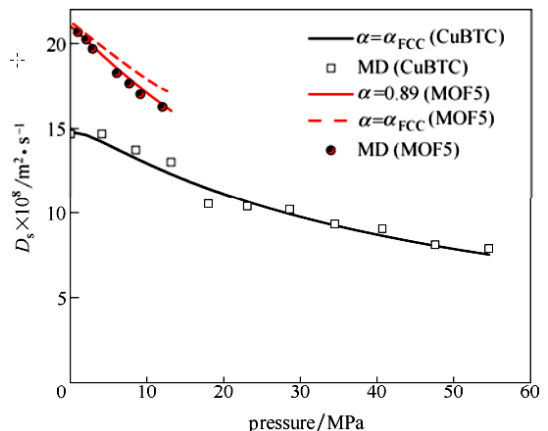

(a)

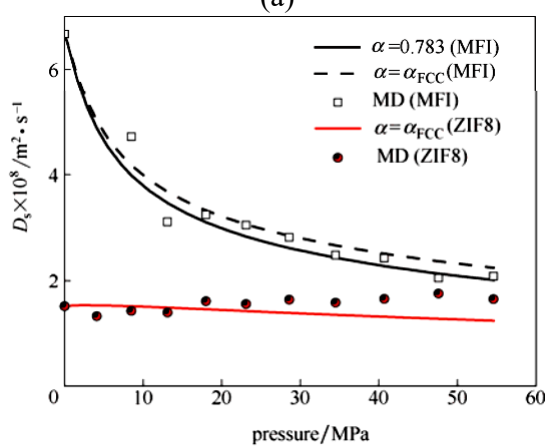

(b)

Fig. 5 Diffusion coefficient of $\mathrm{H}_{2}$ in four types of porous material

\section{Conclusion}

As a high-performance nanoporous material, MOF has a good application prospect. Its adsorption performance prediction is the key to material screening. Due to the limitation of computational speed, the traditional computer simulation has low computational efficiency. The recently developed fast Fourier transform CDFT can solve this problem well. It can greatly improve computational efficiency while keeping actuarial accuracy unchanged. However, CDFT also has some shortcomings. The main problem is how to deal with the adsorption problem of molecules with complex spatial structures, especially when the molecular dipole moment is large. Therefore, the improvement of CDFT theory itself is the key to extend CDFT to more complex systems. Based on the current theory of CDFT, it may be a promising method to predict the behavior of fluid in MOF materials with molecular simulation. In a word, the improvement of CDFT theory and the combination of CDFT and molecular simulation are the two main ways for CDFT to predict gas adsorption in MOF.

\section{Acknowledgments}

This work was supported by the National Key Research and Development Program (No.2017YFC0307800).

\section{References}

1. O.K. Farha, A.O. Yazaydin, Nature Chemistry, 2, 944 (2010)

2. D.W. Siderius, L.D. Gelb, Langmuir, 25, 1296 (2009)

3. Y. Liu, H.L. Liu, Y. Hu, Journal of Physical Chemistry B, 113, 12326 (2009)

4. J. Fu, Y. Liu, Y. Tian, Journal of Physical Chemistry C, 119, 5374 (2015)

5. Y. Liu, S.L. Zhao, H.L. Liu, AIChE Journal, 61(9):2951 (2015)

6. A.I. Skoulidas, D.S. Sholl, Journal of Physical Chemistry B, 106, 5058 (2002)

7. M. Dzugutov, Nature, 381, 137 (1996)

8. Y. Liu, J. Fu, J.Z. Wu, Langmuir, 29, 12997 (2013) 